\title{
Modeling the Spread of Tree Pests after Aerial Pest Control with the Means of a Geo-Information System
}

\author{
Colja Krugmann, Jochen Wittmann*
}

HTW Berlin University of Applied Sciences, Environmental Informatics, Wilhelminenhofstraße 75A, 12459 Berlin, Germany, * wittmann@htw-berlin.de

SNE 31(4), 2021, 223-226, DOI: 10.11128/sne.31.sn.10586 Received: 2021-03-15 (Selected ASIM SST 2020 Postconf. Pub., English version); Revised: 2021-10-15; Accepted: 2021-11-15 SNE - Simulation Notes Europe, ARGESIM Publisher Vienna ISSN Print 2305-9974, Online 2306-0271, www.sne-journal.org

Abstract. As part of a student project for the module Environmental and Geoinformation Systems, this article takes up the discussion about aerial pest control in forests. A model based solely on publicly available data is created to identify the area in which the aerial application of insecticides is permitted. It further estimates the renewed spread of forest pests after the control measure using a simple dynamic model. The background of this model is the fact that the insecticide is applied in compliance with protective zones around populated areas, as well as surface waters and forest edges. In those untreated areas of the forest, the pests survive and will spread anew. Assuming a certain duration for the propagation cycle of the pests as well as an average radius, the dynamic spread of the pest population over several propagation cycles can be simulated and displayed using simple onboard methods of a geoinformation system. The difficulty in determining these zones is the fact that the geodata of German forests is frequently intersected by small roads that are irrelevant to the aerial application of the insecticide. As a feasibility study, this paper proves that such a model can provide useful information. For the case of an actual aerial application of an insecticide, it provides a list of essential parameters for a practically oriented simulation.

\section{Motivation}

In 2019, an aerial application of Syngenta's "Karate Forst flüssig", a liquid agent against forest pests, primarily the nun moth (Lymantria monacha), was considered in Brandenburg. A large number of environmentalists and local residents opposed these plans (rbb24.de, 2019). Pine trees account for $70 \%$ of Brandenburg's forests.

This monoculture in combination with the drought of the recent years increased the susceptibility of the trees to pest organisms such as the pinetree lappet (Dendrolimus pini), pine looper moth (Bupalus piniaria), pine sawfly (Diprion pini), pine beauty moth (Panolis flammea) and nun moth (Lymantria monacha). Forest monitoring predicted a high risk of clearcutting under favorable conditions for the pests, such as warm and dry weather.
Pine trees can survive a onetime defoliation of about $90 \%$ with little loss, but in case of further or repeated damage, the forest is expected to die. As forests are a significant factor in the carbon dioxide $\left(\mathrm{CO}_{2}\right)$ balance, a complete defoliation would transform them from a $\mathrm{CO}_{2}$ sink into a $\mathrm{CO}_{2}$ source.

The Landeskompetenzentrum Forst Eberswalde (LFE) planned to encounter this situation with aerial pest control using Syngenta's Karate Forst flüssig. This insecticide is a contact poison that is applied to the canopy of the infested trees in highly diluted form in order to kill the nun moth in its caterpillar stage (Waldschutzmaßnahmen gegen Nonnenraupen | Landesbetrieb Forst Brandenburg, n.d.). Conservationists strongly doubted the usefulness and environmental compatibility of this approach as the natural enemies of the pests would also be affected (rbb24.de, 2019). Though according to the LFE, the concentration of the insecticide can be set just high enough to kill only the nun moth caterpillars, leaving their natural enemies and for example cockchafers or other ground beetles unaffected (Waldschutzmaßnahmen gegen Nonnenraupen | Landesbetrieb Forst Brandenburg, n.d.).

At this point, the present feasibility study intervenes in the discussion. It examines whether statements about the effects of aerial pest control on the development of pest infestations can be made by means of a model developed based exclusively on publicly available data. In the model special consideration is given to the importance of the safety distances that must be maintained around settlements, water bodies, etc. when applying the insecticide. These protected zones thus constitute refuge areas for the pest populations that can not only recover but also spread anew.

\section{Model Idea and Preparation for a GIS Analysis}

The idea of the present work is first of all to use only publicly available maps as basic data for the usecase showing the areas permitted and suitable for the aerial application of the insecticide. It also attempts to estimate the distribution of forest pests after the control measure within the framework of the development of a dynamic model. 
The basic assumption for the modeling is that the protected zones that are excluded from the application of the pesticide provide refuge areas for the pests and therefore serve as a basis for a subsequent reinfestation. Assuming a certain duration for the reproduction cycle of the pests as well as an average distribution radius, the dynamic spread of the pest population over several reproduction cycles can be simulated and represented using only standard methods for spatial analysis provided by a geographic information system (Figure 1).

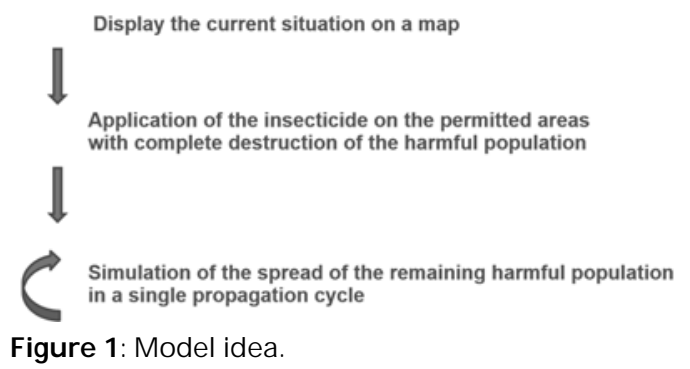

The aim of this modeling is to highlight the influence and the importance of the protected zones and to open them to a quantitative investigation. A topographically highly structured landscape that is strongly interspersed with large and numerous areas protected from the application of the insecticide will show very different effects on the assumed reinfestation process than a landscape with large structures with accordingly less protected areas.

To carry out this analysis in a geoinformation system, the following subtasks arise for the modelling:

1. finding and processing suitable publicly accessible geodata

2. determination of the parameter values necessary for modelling

a. With regard to the application instructions

of the insecticide

i. Safety distance to settlements

ii. Safety distance to bodies of water

iii. Safety distance to forest edges

b. With regard to the biological data of the pest

i. Duration of a reproduction cycle

ii. Propagation range in one reproduction cycle

\section{Analysis Workflow}

\subsection{Step 0: Creating a Base Map and Basic Geodata}

The limitation of this feasibility study to publicly available maps represents a certain challenge, which on the one hand requires additional work steps, but on the other hand underlines the independence of this approach from already existing, but only limited and/or conditionally available data.
A region in Brandenburg between Bad Belzig and Werder (Havel) was chosen as the sample area. The underlying geodata for the modeling is as following:

- A cutout from openstreetmap was selected as base (Geofabrik Download Server, 2019).

- The watercourse data as shape files originate from Geoportal Brandenburg (Geoportal Brandenburg, 2019).

- The road network data for Brandenburg was downloaded as GML-file from Inspire Brandenburg via API. (LGB (Landesvermessung und Geobasisdaten Brandenburg), 2019).

- The forest data originate from the Landeskompetenzzentrum Brandenburg and were available as a GML file (Forstgrunddaten - Flaechen - Waldbedeckung - Land Brandenburg INSPIRE, 2018)

- Ideally, residential and agricultural areas are available as shape files to apply the exact safety distances to each. In this example they are represented by the blank spaces within the forest areas.

\subsection{Step 1: Creating Contiguous Forest Areas}

The geodata of the forest areas are dissected by smaller or dirt roads, thus representing fictitious edges of the forests that would lead to protected zones in the modeling. With respect to an insecticide application, forest roads do not count as a boundary of a forest area and must therefore be ignored for this project.

To achieve this a buffer is placed around the forest areas to cover the respective forest roads. The resulting overlapping forest polygons are then merged by applying a dissolve. A subsequent negative buffer of the same width is applied to the resulting areas to reduce them to their original and actual size. (Sketch of the procedure Figure 2, base map after this processing step in Figure 3).

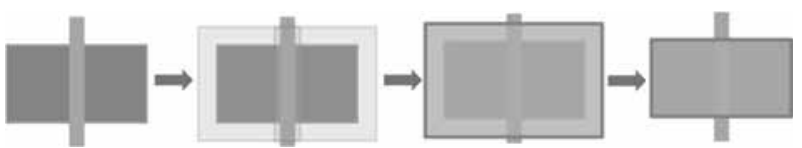

Figure 2: Step 1: Creating contiguous forest areas.

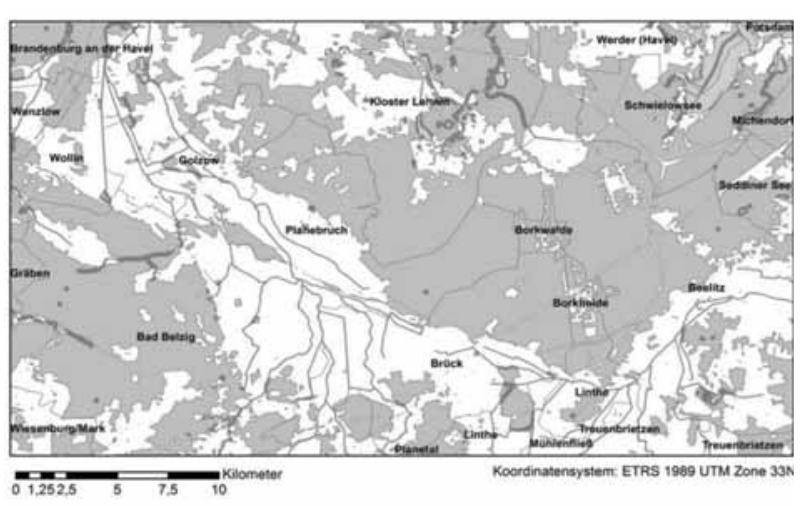

Figure 3: Base map after step 1. 


\subsection{Step 2: Creating Uniform Water Surfaces and their Protected Zones}

Geodata for lakes are available as polygons, whereas rivers and canals are represented by lines. To obtain one layer containing all waterbodies as continuous polygons, the lines representing rivers and creeks need to be converted into polygons. They can subsequently be combined with the lake areas.

For this purpose, a buffer was created around the rivers as a line feature. Afterwards lakes and rivers can be merged to one layer "water bodies", which is relevant for the determination of the protected zones. All water features must be buffered again by the width of the protected zone to create polygons that represent the water bodies including their safety distance (Figure 4).

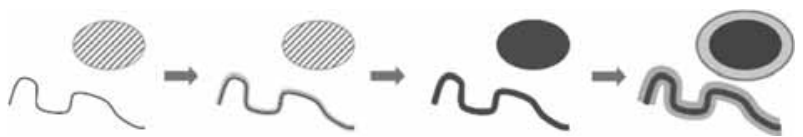

Figure 4: Step 2: Creating uniform water surfaces with safety distance.

\subsection{Step 3: Creating the Safety Distance from Major Roads and Merging of all Protected Zones}

The aerial application of pesticides over larger roads, such as federal and district roads or federal motorways, is not permitted. As for rivers, geodata for roads are represented as line features. To determine the protected zones along these roads, they are buffered according to an averaged road width and the prescribed safety distance. The resulting protected zones around roads are then merged with those around water bodies. (Figure 5).

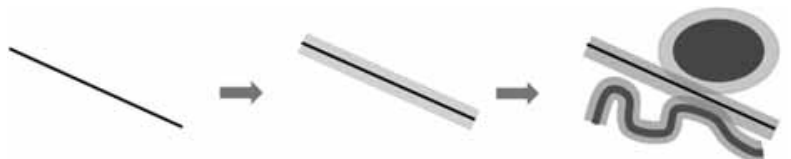

Figure 5: Step 3: Safety distance from major roads and merging of protected zones.

\subsection{Step 4: Determining all Forest Areas left Untreated by the Aerial Pest Control}

To determine all forest areas that must not be treated with the insecticide, the treatable forest area must be identified. This is accomplished by applying a negative buffer of the prescribed safety distance to forest edges to forest polygons resulting from step 1 . This area is then intersected with the polygons representing the protected zones of water bodies and major roads from steps 2 and step 3. The result of this intersection is now subtracted from the buffered forest area by a symmetrical difference, to obtain all the areas of the forest eligible for an aerial application of the insecticide.
Another symmetrical difference of this area and the entire forest area results in a polygon representing all forest areas excluded from the aerial application (Figure 6).

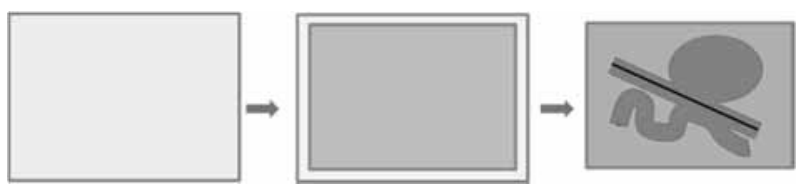

Figure 6: Step 4: Determination of untreated forest areas.

\subsection{Step 5: Spread of the Pest}

It is assumed that the pests survive completely in the protected areas. From there they can then spread again to the neighboring and previously treated areas. This spreading process is modeled a buffer around the untreated areas. The buffer width corresponds to the radius of the spread of the pest in one propagation cycle. This assumed spread extends beyond the forest boundaries. Accordingly, the resulting polygons are intersected with the forest areas resulting from step 1 . In addition, there may be an overlap of parts of the dispersal zone, which are removed by a dissolve (Figure 7). The result is a polygon representing all infested areas including the protected zones from step 4 .

This procedure can be run repeatedly based on these polygons to simulate the expansion of the pest with each propagation cycle.

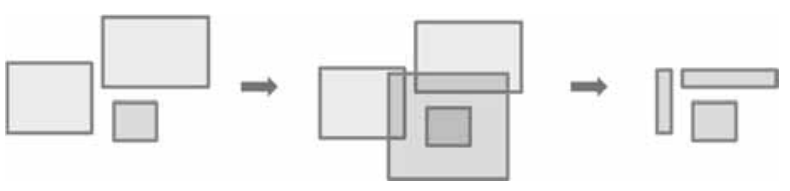

Figure 7: Step 5: Spread of the pest.

\section{Results}

\subsection{Feasibility Study for the Sample Region}

The analysis described in the previous section were applied to the sample region from Figure 3 by using the Model Builder in Esri's ArcMap 10.5. The values for the various parameters mentioned in the individual steps were plausibly estimated or extracted from manuals for the aerial application of pesticides. The exact values are deliberately not mentioned in this paper, since the model is only a prototype aimed at the analysis workflow. The results can serve as a plausibility test. The parameterization will be discussed separately in the following subsection.

As a result of the modeling and the simulation, three qualitative conclusions can be drawn:

1. The described step-by-step analysis workflow can be mapped completely and transparently with the standard methods of ArcMap. It is available as a freely parameterizable new tool. 
2. It is possible to implement a simple dynamic simulation by iterating the propagation method explained in step 5.

3. The procedure described by the workflow leads to plausible results, as shown in Figure 8.

As shown in the result map after three defensively parameterized propagation cycles large parts of the forest area are already newly colonized by the pest (marked orange to yellow, the protected zones are marked red in Figure 8 ). The model emphasizes the dependency of the degree of pest infestation on the landscape's structure.

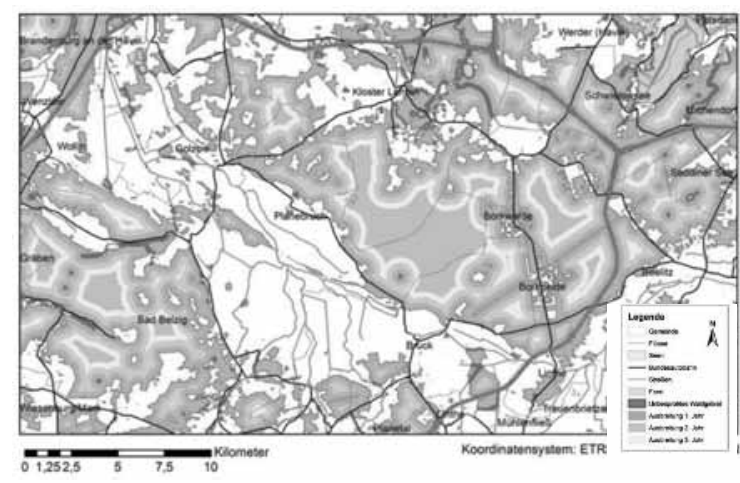

Figure 8: Simulated state of pest infestation for the example region after three propagation cycles.

\subsection{Necessary Parameter Values for an Accurate Model Study}

As already mentioned, the model presented is based on publicly available data. In several instances it is dependent on parameter values that could only be plausibly estimated in the feasibility study (e.g. the width of roads and rivers). For a reliable quantitative investigation, the parameters of the model must be filled with valid values. However, it should be noted that an aim of this specific modeling was to obtain results using a small number of parameters, that are relatively easy to determine.

Below is the complete list of these model parameters that must be provided for a concrete application:

1. Geospatial data:

a. Base map (the feasibility study deliberately works exclusively with publicly available material; better suited thematic base maps may be available).

b. Width of small watercourses

c. Width of roads

2. Application instructions of the insecticide:

a. Minimum distance to forest edges

b. Minimum distance to surface water

c. Minimum distance to residential areas

3. Data on the biology of the pest:

a. Duration of a reproductive cycle

b. Range of spread in one propagation cycle.
It goes without saying that many other influencing factors are conceivable and useful for a more detailed modeling. Two examples for influencing factors on the risk and spread of a pest infestation that were ignored in this model are the weather and the forest's composition as its main goal was to demonstrate its feasibility.

\section{Conclusion}

The present work is intended to show that even a very simple modelling approach can generate meaningful results allowing for a determination of untreated areas, as well as for an estimation of the effects of an intervention in pest infestations by aerial application insecticide. With a manageable amount of parameter values required and little expenditure, both algorithmically and in terms of software technology, this model can help objectifying a discussion about the application of insecticides by investigating different scenarios obtained through parameter variations.

On the methodological level, the presented model shows that the frequently discussed coupling and/or integration of geoinformation and simulation systems can succeed pragmatically and successfully for individual projects with a narrow requirement profile on the one hand and a very pragmatic modeling approach on the other.

\section{References}

[1] Geofabrik.de. 2019. Geofabrik Download Server. [online] Available at: 〈https://download.geofabrik. de/europe.html> [Accessed 7 July 2019].

[2] Landesbetrieb Forst Brandenburg. n.d. Waldschutzmaßnahmen gegen Nonnenraupen | Landesbetrieb Forst Brandenburg. [online] Available at: <https://forst. brandenburg.de/lfb/de/lfe/waldschutzinformationen/waldschutzmassnahmen-gegen-nonnenraupen/> [Accessed 3 April 2020].

[3] Brandenburg-forst.de. 2018. Forstgrunddaten - Flaechen Waldbedeckung - Land Brandenburg INSPIRE. [online] Available at: $<$ http://www. brandenburg-forst.de/inspire/dls/ifgk_wld/> [Accessed 23 April 2018].

[4] LGB (Landesvermessung und Geobasisdaten Brandenburg). 2019. Geoportal Brandenburt. [online] Available at: <https://geoportal. brandenburg.de/ startseite/> [Accessed 14 May 2019].

[5] Majunke, C., Möller, K. and Funke, M., 2004. Die Nonne. Waldschutzmerkblatt 52. [online] Potsdam: Landesforstanstalt Eberswalde. Available at: <https://opus4.kobv.de/opus4-slbp/files/5068/ nonne.pdf> [Accessed 1 May 2019].

[6] OpenStreetMap. 2019. OpenStreetMap. [online] Available at: <https://www.openstreetmap.org> [Accessed 28 April 2019].

[7] rbb24.de, 2019. Mit „Karate Forst“ gegen Raupen in Brandenburgs Wäldern. [online] Available at: <https://www.rbb24.de/panorama/beitrag/2019/04/brandenburg-beelitz-mit-karate-forst-fluessig-gegen-insekten.html> [Accessed 3 April 2020]. 